\title{
Effects of Bridging Exercise using Vibration Stimulation and ADIMs on the Lengthening and Thickness of Transversus Abdominis in Healthy Adults
}

\author{
Kwan-Woo Kong \\ Graduate School of Rehabilitation Science, Daegu University, Gyeongsan, Korea
}

\begin{abstract}
Purpose: This study was conducted in an effort to determine the effects of various abdominal drawing-in maneuver (ADIM) on the thickness and length of the transversus abdominis (TrA) when using lumbar stabilization exercises on healthy adults.

Methods: 72 healthy adults were divided into four groups of 18 subjects each, to which different ADIM methods were applied. 1) a simple ADIM exercise, 2) an ADIM with pressure bio-feedback units, 3) an ADIM exercise with sling, and 4) an ADIM exercise with sling and vibration. Changes in the thickness and sliding length of TrA were measured when ADIM was conducted in the supine position prior to exercise and again when beginning the exercises. Following exercise, changes in the thickness and sliding length of TrA were measured using the same methods. Differences in group measurements prior to and following exercise were compared using a one-way analysis of variance. A paired t-test was applied to compare the before and after differences within each group.

Results: Differences in TrA thickness change revealed that the ADIM exercise with sling and vibration group showed a significant difference in measurements taken prior to and following exercise. Differences in TrA length change revealed that the ADIM exercise with sling and vibration group showed a significant difference in measurements taken prior to and following exercise.

Conclusion: ADIM exercise with vibration stimulation conducted in the bridge posture while in a prone position using a sling can be recommended as an effective exercise to improve the function of lumbar TrA.
\end{abstract}

Keywords: Vibration, Transversus abdominis, Sling

\section{INTRODUCTION}

In recent years, a number of treatment methods for lower back pain have been developed. Among them, an abdominal drawing-in maneuver (ADIM) method has been proposed based on the concept of spinal stabilization. Hodges et al. ${ }^{1}$ experimented with biofeedforward mechanisms of trunk in relation to functional movements in the upper extremities; experiment results indicated that the contraction of the transversus abdominis ( $\operatorname{Tr} \mathrm{A})$ occurred regardless of the direction of movement in the upper extremities and that the contraction time of TrA was delayed in patients with lower back pain due to the presence of a bio-feedforward mechanism. The ADIM method is used only to contract $\operatorname{Tr} A$ and the internal

Received Nov 18, 2016 Revised Dec 2, 2016

Accepted Dec 8, 2016

Corresponding author Kwan-Woo Kong

E-mail kanoo75@naver.com oblique abdominal muscle (IO) in an effort to increase intra-abdominal pressure by pulling the abdominal wall inward. The bridge exercise with ADIM was more efficient for increasing balance ability in patients with stroke. ${ }^{2}$ Abdominal muscle activity, in combination with the ADIM, affected the grip strength, positively. ${ }^{3}$

Monica et al. ${ }^{14}$ suggested that a lateral $\operatorname{Tr} A$ slide in patients with chronic lower back pain is an effective means of pain reduction. Using magnetic resonance imaging and real-time ultrasounds, Hides et al. ${ }^{15}$ studied a function of motion that occurs when TrA pulls at the abdominal wall. They reported that when TrA pulls at the abdominal wall, it acts as a sort of corset, increasing thickness and lateral slide and enhancing the stability of one's waist and pelvis.

A sling exercise using a swing rope for lumbar stabilization can

Copylight (C2016 The Korea Society of Physical Therapy

This is an Open Access article distribute under the terms of the Creative Commons Attribution Non-commercial License (Http:// creativecommons.org/license/by-nc/4.o.) which permits unrestricted non-commercial use, distribution, and reproduction in any medium, provided the original work is properly cited. 
achieve active closed kinetic chain exercise while minimizing gravitational resistance; this can increase efficiency in stability, proprioceptive integrated senses, and motor control. ${ }^{4}$ One method used to increase the effects of sling exercise is the application of vibration during exercise. ${ }^{5}$ To increase muscle strength and muscle mass, vibrations of 25-45 Hz frequency are used. ${ }^{6}$

Many exercises have been introduced to activate local muscles. However, among the many exercise methods, there is a lack of research on the activation of local muscle accompanied by vibrations in the sling, and also a comparison study on slip of the TrA muscles during activation of local muscles. Therefore, the method of activation of local muscle through various existing ADIMs and the ADIM methods using sling and vibration will provide a more efficient exercise method by comparing and analyzing each method. The ADIM method using sling and vibration will be based on muscle bilization and exercise effects.

The present study aims to determine the effects of the following methods on the thickness and length of TrA: 1) a simple ADIM exercise gruop, 2) an ADIM with pressure bio-feedback units (PBU) group, 3) an ADIM exercise with sling group, and 4) an ADIM exercise with sling and vibration group.

\section{METHODS}

\section{Subjects}

To complete the present study, 72 healthy adults whose mean age, mean height, and mean weight were $22.5 \pm 2.01$ years, $167.95 \pm 6.51$ $\mathrm{cm}$, and $62.5 \pm 8.60 \mathrm{~kg}$, respectively, were recruited. The selection criteria for the subjects were as follows: 1) subjects had no history of disease of the nervous or musculoskeletal systems and were without functional disability, and 2) no regular or systematic exercise was performed by subjects at the time of their selection. All subjects gave voluntary consent to participate in the experiment upon being fully informed about the purpose and methods of the study. The present study was approved by the Daegu University Institutional Review Board in accordance with the ethical principles of the Declaration of Helsinki.

\section{Experimental methods}

\section{1) Measurement}

Subjects $(n=72)$ were randomly divided to four groups $(n=18)$.
All Subjects were instructed to bend their knees at $90^{\circ}$ prior to and following the experiment, and a PBU was placed below their waists while in a supine position. Then, subjects performed ADIM via verbal instruction, and the resulting ultrasonic waves (Terason $\mathrm{t} 3000$ Ultrasound System, Apple, America) were measured. A mean value was derived after three repeated measurements, with participants being granted one minute of rest time between measurements. A single evaluator measured all subjects in order to minimize errors that may arise due to inter-rater differences. Ultrasound gel was applied to a probe and to the participants' skin, and the thickness and length of TrA muscle was measured at the same position; this position was located $1 \mathrm{~cm}$ away from the boundary that separates from the fascia in the center of the muscle. ${ }^{9}$ The anterolateral muscles were also measured, which were located on the center axilla line between the iliac crest and the inferior angle of the costa. ${ }^{10-13}$ To measure the sliding length of $\operatorname{TrA}$, measurements were taken while subjects performed the exercises via a fixed probe that was placed on the boundary of the TrA fascia and displayed at the left end of the ultrasound measuring screen.

\section{(1) Simple ADIM exercise group}

Subjects in the simple ADIM exercise group were instructed to bend their knees at $90^{\circ}$, lie in a supine position, ${ }^{7}$ and perform ADIM exercises via verbal instruction. The ADIM exercises consisted of three sets; in each set, subjects maintained their position for $12 \mathrm{sec}-$ onds. This process was repeated ten times. A rest period of $30 \mathrm{sec}-$ onds was granted during each set.

\section{(2) ADIM with PBU group}

Subjects in the ADIM with PBU (stabilizer, Chattanooga, USA) group were instructed to bend their knees at $90^{\circ}$ and lie in a supine position. A PBU was then placed below their waists, and subjects were instructed to maintain ADIM for 12 seconds. This process was repeated 10 times while subjects maintained $40 \mathrm{mmHg}$, and three sets of the above exercise were performed.

\section{(3) ADIM exercise with sling group}

Subjects in the ADIM exercise with sling (sling exercise tool, Red$\operatorname{cord}^{\circledR}$, Norway) group conducted the abovementioned exercise using a sling. ADIM was performed using a method that induced the contraction of TrA by contracting the urethra at the prone lumbar 
position via a sling that used an elastic cord. ${ }^{8}$ A single exercise set consisted of maintaining this position for 120 seconds; each subject conducted three sets.

\section{(4) ADIM exercise with sling and vibration group}

Subjects in the ADIM exercise with sling and vibration group were conducted ADIM by applying a partial vibration of $30 \mathrm{~Hz}$ frequency using a Redcord vibration tool (Stimula Redcord ${ }^{\circledR}$, Norway) while exercising in the same method as the second experimental group. A single exercise set consisted of maintaining this position for 120 seconds; again, each participant conducted three sets.

The data were subjected to the shapiro-wilk test to check the normal distribution, and the normal distribution was confirmed and the parameter test was performed. Data analysis was conducted using SPSS 18.0 for Windows to determine whether differences existed between the experimental and control groups with regard to gender, age, height, and weight. One-way ANOVA was used to compare the thickness and length of TrA as well as to detect differences in muscle activity between the various groups prior to and following the experiment. For post-hoc analysis, a least significant difference (LSD) procedure was employed. Additionally, a paired ttest was used to compare differences within each group prior to and following the experiment. The statistical significance a was set to 0.05 .

\section{RESULTS}

In the ADIM exercise group with sling and vibration, a significant difference in changes to the thickness of TrA was found via ultrasound imaging prior to and following the exercises $(\mathrm{p}<0.05)$ (Table 1). In this group, a significant difference in changes to the length of TrA was also found via ultrasound imaging prior to and following the exercises $(\mathrm{p}<0.05)$. In comparing the results of the various groups, a significant difference was found in these values difference $(\mathrm{p}<0.05)$. Post-hoc analysis results and the comparison of changes in TrA length revealed a significant difference between the ADIM with sling and vibration group and the other groups. A more slide of TrA was found in the group that performed the

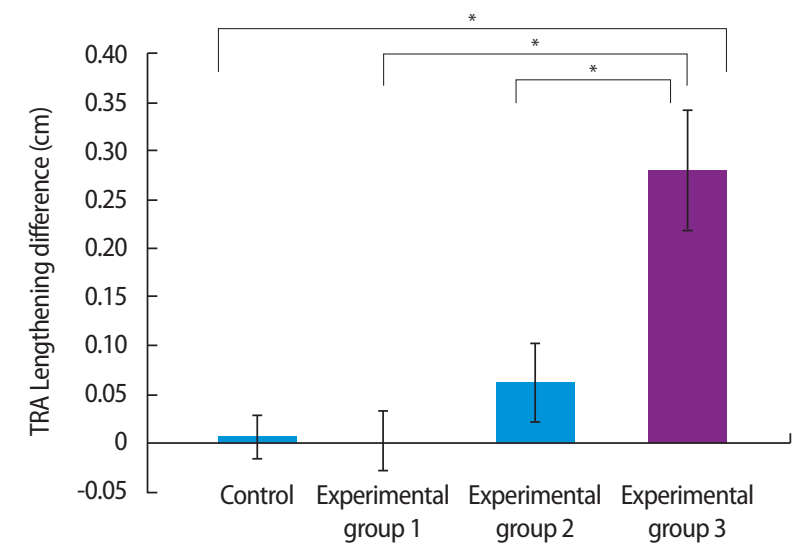

Figure 1. Comparison of changes in TRA muscle lengthening difference.

Table 1. Comparison of changes in TRA muscle thickness (unit: $\mathrm{cm})(\mathrm{n}=72$ )

\begin{tabular}{|c|c|c|c|c|}
\hline & Pre & Post & Difference & $\mathrm{t}$ \\
\hline ADIM exercise group $(n=18)$ & $0.15 \pm 0.02$ & $0.13 \pm 0.02$ & $-0.01 \pm 0.01$ & 0.77 \\
\hline ADIM exercise with pressure biofeedback unit gruop $(n=18)$ & $0.12 \pm 0.01$ & $0.14 \pm 0.02$ & $0.02 \pm 0.01$ & -1.43 \\
\hline ADIM exercise with sling group $(n=18)$ & $0.13 \pm 0.01$ & $0.14 \pm 0.01$ & $0.01 \pm 0.02$ & -0.63 \\
\hline ADIM exercise with sling \& vibration group $(n=18)$ & $0.11 \pm 0.01$ & $0.15 \pm 0.02$ & $0.04 \pm 0.01$ & $-2.73^{*}$ \\
\hline Difference F & \multicolumn{4}{|c|}{$1.92(p=0.135)$} \\
\hline
\end{tabular}

ADIM: various abdominal drawing-in maneuver. ${ }^{*} \mathrm{p}<0.05$.

Table 2. Comparison of changes in transversus abdominis muscle lengthening (unit: $m m)(n=72)$

\begin{tabular}{|c|c|c|c|c|}
\hline & Pre & Post & Difference & $\mathrm{t}$ \\
\hline ADIM exercise group $(n=18)$ & $8.81 \pm 0.57$ & $8.29 \pm 0.57$ & $0.07 \pm 0.22$ & -0.37 \\
\hline ADIM exercise with pressure biofeedback unit gruop $(n=18)$ & $8.86 \pm 0.73$ & $8.88 \pm 0.61$ & $0.03 \pm 0.32$ & -0.07 \\
\hline ADIM exercise with sling group $(n=18)$ & $9.29 \pm 0.47$ & $9.90 \pm 0.535$ & $0.62 \pm 0.40$ & -1.52 \\
\hline ADIM exercise with sling \& vibration group $(n=18)$ & $8.94 \pm 0.58$ & $11.73 \pm 0.51$ & $2.79 \pm 0.63$ & $-4.45^{*}$ \\
\hline Difference $\mathrm{F}$ & \multicolumn{4}{|c|}{$9.70(p=0.01)^{\star}$} \\
\hline
\end{tabular}

ADIM: various abdominal drawing-in maneuver. ${ }^{*} p<0.05$. 
ADIM with sling and vibration than was found in the other groups $(\mathrm{p}<0.05)$ (Table 2) (Figure 1).

\section{DISCUSSION}

The experiment results indicated that a significant difference in changes to the thickness of TrA prior to and following exercise existed in the ADIM exercise group with sling and vibration. In comparing the differences in TrA length between groups, a significant increase in changes to TrA length was found in the ADIM exercise group with sling and vibration.

The present study revealed a greater difference in the changes to length that occur due to increased sliding in the group that used both a sling and a vibration. Until today, most studies of TrA activation have measured the thickness of TrA. The present study, however, has verified that in addition to the existing methods, lateral slide can also be an important assessment method. Furthermore, it is necessary to conduct additional studies of methods that can be used to alter the length of TrA such methods may utilize a stabilization of the waist or pelvis in patients with lower back pain.

Critchley and Coutts ${ }^{16}$ reported that an experimental group that performed ADIM in the quadruped position discovered an increased thickness of TrA when measuring that factor via real time ultrasounds. Shi et al. ${ }^{17}$ reported that when muscles were active, muscle thickness increased. Thus, an increase in the thickness of TrA can be interpreted as an increase in muscle activity. The present study showed that the ADIM exercise group with sling and vibration demonstrated a significant increase in the thickness of TrA following ADIM exercise. However, no significant difference was found in the other ADIM exercise groups prior to or following the exercise. This was because groups whose members performed simple ADIM movements or ADIM in conjunction with PBU had a shorter exercise time of seven to eight minutes, which did not lead to any significant difference. Similarly, the ADIM with sling group showed no significant effects on the thickness of TrA. Therefore, exercise with sling and vibration as an exercise method for immediate effect within a short time can be effective.

In this study, the vibration stimulus was used to easily set the frequency using a local stimulator. Vibration represents a strong stimulus for musculoskeletal structures due to the need to quickly modulate muscle stiffness to accommodate the vibratory waves. This re- sponse is mediated by monosynaptic and polysynaptic afferent pathways, which are capable of triggering specific hormonal responses. It appears that a subsequent voluntary activation can be performed with central and peripheral structures in an elevated excitatory state. ${ }^{18}$ In the study of the lumbar deep muscle using magnetic resonance imaging, 20 healthy male subjects underwent weight bearing exercise with 6 weeks full body vibration. The result of magnetic resonance imaging showed that the cross-sectional area of multifidus atrophy was significantly decreased. ${ }^{19}$ In this study, it was concluded that the TrA muscles of the ADIM with sling and vibration group also showed significant changes because the specific frequency of vibration stimulated the target muscle to increase the voluntary activity.

In their study of deep cervical flexor, Yun ${ }^{20}$ reported that vibration stimulation added to closed chain exercise of the neck is thought to be effective in improving the functions and muscle strength and muscle endurance of the deep cervical flexor. The present study revealed a significant difference in the thickness and length of TrA prior to and following exercise in the ADIM exercise group with sling and vibration. A significant difference was also found in the length of TrA not only before and after exercise but also in the comparison between groups. Previous studies ${ }^{20}$ have reported significant results of fine motor activation by vibration stimulation; the present study led to a similar result of increased fine motor activation following the application of vibration. Based on the above results, exercises performed in conjunction with vibration stimulation in the bridge posture while in a prone position and while using a sling can be recommended as an effective means of improving the function of lumbar TrA.

The present study has the following limitations: the study result cannot be generalized to all patients with lower back pain, as it was conducted in normal healthy adults. Furthermore, although the present study verified immediate changes in the thickness and length of TrA by cross-section study design, we cannot know how long this effect may last. Thus, it is necessary to determine which changes would occur if these exercises were applied three to four times per week. To this end, future research should examine how long these effects might last.

\section{REFERENCES}

1. Hodges PW, Richardson CA. Inefficient muscular stabilization of the 
lumbar spine associated with low back pain: a motor control evaluation of trasversus abdominis. Spine. 1996;21(22):2640-50.

2. Song GB, Heo JY. The effects of bridge exercise with abdominal drawingin on balance in patients with stroke. J Kor Phys Ther. 2016;28:1-7.

3. Lee M. Effect of abdominal muscle activity in combination with the abdominal drawing-in maneuver for grip strength in healthy young adults. J Kor Phys Ther. 2012;24:1-6.

4. Yun K, Lee S, Park J. Effects of closed chain exercises for the lumbar region performed with local vibration applied to an unstable support surface on the thickness and length of the transverse abdominis. J Phys Ther Sci. 2015;27(1):101-3.

5. Kim ER, Oh JS, Yoo WG. Effect of vibration frequency on serratus anterior muscle activity during performance of the push-up plus with a redcord sling. J Phys Ther Sci. 2014;26(8):1275-6.

6. Prisby RD, Lafage-Proust MH, Malaval L et al. Effects of whole body vibration on the skeleton and other organ systems in man and animal models: what we know and what we need to know. Ageing Res Rev. 2008;7(4):319-29.

7. Richardson CA, Jull G. Muscle control-pain control. what exercise would you prescribe. Man Ther. 1995;1(1):2-10.

8. Sapsford RR, Hodges PW. Contraction of the pelvic floor muscles during abdominal maneuvers. Arch Phys Med Rehabil. 2001;82(8):1081-8.

9. Mannion AF, Pulkovski N, Gubler D et al. Muscle thickness changes during abdominal hollowing: an assessment of between-day measurement error in control sand patients with chronic low back pain. Eur Spine J. 2008;17(4):494-501.

10. Koppenhaver SL, Hebert JJ, Fritz JM et al. Reliability of rehabilitative ultrasound imaging of the transversus abdominis and lumbar multifidus muscles. Arch Phys Med Rehabil. 2009;90(1):87-94.

11. Childs JD. The use of ultrasound imaging of the abdominal drawing-in maneuver in subjects with low back pain. J Orthop Sports Phys Ther. 2005;35(6):34.

12. McMeeken JM, Beith LD, Newham DJ et al. The relationship between EMG and change in thickness of transversus abdominis. Clin Biomech. 2004;19(4):337-42.

13. Whittaker JL. Ultrasound imaging of the lateral abdominal wall muscle in individuals with lumbopelvic pain and sign of concurrent hypocapnia. Man Ther. 2008;13(5):404-10.

14. Monica UT, Tom ILN, Jon M et al. Is activation of transversus abdominis and obliquus internus abdominis associated with long-term changes in chronic low back pain? a prospective study with 1-year follow-up. Br J Sports Med. 2012;46(10):729-34.

15. Hides JA, Wilson SJ, Stanton W et al. An MRI investigation into the function of the transversus abdominis muscle during "drawing-in" of the abdominal wall. Spine. 2006;31(6):175-8.

16. Critchley DJ, Coutts FJ. Abdominal muscle function in chronic low back pain patients: measurement with real-time ultra sound scanning. Physiotherapy. 2002;88(6):322-32.

17. Shi J, Zheng YP, Chen X et al. Assessment of muscle fatigue using sonomyography: muscle thickness change detected from ultrasound images. Med Eng Phys. 2007;29(4):472-9.

18. Cardinale M, Bosco C. The use of vibration as an exercise intervention. exercise and sport sciences reviews. 2003;31(1):3-7.

19. Belavý DL, Hides JA, Wilson SJ et al. Resistive simulated weight bearing exercise with whole body vibration reduces lumbar spine deconditioning in bed-rest. Spine. 2008;33(5):E121-31.

20. Yun KH. The effects of closed chain exercise of the neck using vibrations on the thicknesses of normal adults. Daegu University. Dissertation of Doctorate Degree. 2014. 